\title{
BMJ Open Cross-cultural adaptation and validation of the caregiver contribution to self-care of chronic illness inventory in China: a cross-sectional study
}

\author{
Dan Dan Chen, ${ }^{1}$ Hui Zhang, ${ }^{1}$ Nianqi Cui $(1),{ }^{2}$ Leiwen Tang, ${ }^{1}$ Jing Shao, ${ }^{3}$ \\ Xiyi Wang (D) , ${ }^{4}$ Dan Wang, ${ }^{1}$ Na Liu, ${ }^{5}$ Zhihong Ye (D) ${ }^{1}$
}

To cite: Chen DD, Zhang $\mathrm{H}$, Cui N, et al. Cross-cultural adaptation and validation of the caregiver contribution to self-care of chronic illness inventory in China: a crosssectional study. BMJ Open 2021;11:e048875. doi:10.1136/ bmjopen-2021-048875

- Prepublication history for this paper is available online. To view these files, please visit the journal online (http://dx.doi org/10.1136/bmjopen-2021 048875).

$\mathrm{DDC}$ and $\mathrm{HZ}$ are joint first authors.

Received 12 January 2021 Accepted 26 July 2021

D Check for updates

(C) Author(s) (or their employer(s)) 2021. Re-use permitted under CC BY-NC. No commercial re-use. See rights and permissions. Published by BMJ.

For numbered affiliations see end of article.

Correspondence to

Dr Zhihong Ye; yezh@zju.edu.cn

\section{ABSTRACT}

Objective Caregiver contribution (CC) is important for the self-care behaviors of chronic disease individuals, as it could enhance patient outcomes. Therefore, it is necessary to assess this CC by using a good validity and reliability instrument. The Caregiver Contribution to SelfCare Chronic IIIness Inventory (CC-SC-CII) was designed to assess CC to self-care behaviors of patients with chronic illness in Italy. However, it was unclear whether this tool had sound psychometrics properties in the context of Chinese culture. Therefore, we performed the cross-cultural adaption of the CC-SC-Cll and we tested its psychometric properties among Chinese caregivers of patients with chronic disease.

Design A cross-sectional observational design.

Settings Participants were recruited from communities and institutions in Pingdingshan, Henan Province, China. Participants 301 caregivers of care recipients with chronic disease completed the Chinese version of the $\mathrm{CC}$ SC-CII (C-CC-SC-CII).

Primary and secondary outcome measures The content validity index of items (I-CVI), the scale content validity index-average (S-CVI/Ave), exploratory factor analysis, confirmatory factor analysis (CFA), internal consistency and item analysis were tested.

Results The range of I-CVI was between 0.833 and 1.00 , and the score of S-CVI was 0.991 . In CFA, the C-CC to selfcare monitoring scale had satisfactory fit indices. However, the C-CC to self-care maintenance and management scales had unsupported fit indices. The reliability coefficients of C-CC-SC-CII were $0.792,0.880$ and 0.870 for its three scales. Item-total correlations were all over 0.590 . Test-retest reliability showed that the range of intraclass correlation coefficients was from 0.728 to 0.783 . Conclusion The C-CC-SC-CII has sound psychometrics characteristics and is a culturally appropriate and reliable instrument for assessing CC to the self-care behaviours of patients with chronic disease in China.

\section{INTRODUCTION}

Chronic diseases are prevalent across both developed and developing countries. It has been estimated that prevalence of chronic diseases will increase by $40 \%$ by $2050 .^{1}$ Furthermore, as healthcare improves and
Strengths and limitations of this study

- Evidence is limited on the caregiver contribution (CC) level to self-care behaviours of individuals with chronic illness in China; this study assessed this issue in over 300 Chinese caregivers.

- The original CC to Self-Care Chronic IIIness Inventory was successfully translated and cross culturally adapted from English to Chinese.

- The caregivers were recruited by employing a convenience sampling and were mainly from one province; hence, the representative of the findings was limited in China.

- Another limitation was that we did not perform concurrent validity.

life expectancy increases, many people will suffer from one or more chronic diseases. ${ }^{2}$ The number of people with multimorbidity may increase by over $1 \%$ per year by $2030 .{ }^{3}$ Chronic diseases caused individuals to experience physical and psychological symptoms. ${ }^{45}$ Moreover, chronic diseases were associated with high morbidity and disability ${ }^{6} 7$ and accounted for over $80 \%$ of all healthcare expenditures, ${ }^{8}$ which resulted in a heavy social and economic burden. In view of the negative outcomes of chronic disease, healthcare professionals and researchers are focusing on how to manage chronic diseases to prevent their deterioration and progression.

De Maria et at proposed that managing chronic illnesses required mastering self-care, which was fundamental in managing chronic diseases. ${ }^{10}$ In the middle-range theory of selfcare of chronic illness, self-care refers to a process of maintaining health through health promotion and disease management. ${ }^{11}$ Researches showed that self-care was conducive to enhancing health-related quality of life in patients with chronic diseases ${ }^{12}$ and reducing mortality. ${ }^{13}$ Nevertheless, due to 
complicated physiological and psychological factors, selfcare behaviours of patients with chronic diseases are not adequate. In addition, with the increase in comorbidities, the self-care of patients decreased. ${ }^{14}$ The inadequate selfcare was related to more emergency department visits and hospitalisations. ${ }^{15} 16$ Some patients with chronic conditions reported that it was difficult for them to take care of themselves due to health conditions, and they sought help and support from others. ${ }^{17}$ Therefore, in addition to self-care, care from others was also critical. ${ }^{18}$

The caregivers include formal caregivers (such as nurses or midwives) and informal caregivers (such as relatives, friends or neighbours). Because of the shortage of care institutions and nursing resources, informal caregivers are needed. Especially, due to the culture of filial piety in China, it is estimated that over $99 \%$ of the daily care for chronic illness is carried out by the patients and family caregivers. ${ }^{19}$ Informal caregivers mainly assist care recipients in symptom and medication management and provide them with daily activity assistance and emotional supports. ${ }^{20}$ The results of qualitative and quantitative research found that informal caregiver contribution (CC) to the self-care behaviours of patients with heart failure could improve patient outcomes. ${ }^{21} 22$ Researches showed that informal care partners played a key role in palliative care and breathlessness care. ${ }^{23}{ }^{24}$ Therefore, the CC should not be underestimated. Given the important role of informal caregivers in Chinese culture, it is necessary to measure the informal CC to self-care of patients with chronic disease in China. Thence, it is also important to develop a good validity and reliability instrument.

There are several tools that could be employed to assess the informal CC to self-care behaviours, including the Caregiver Contribution to Self-Care in Ostomy Patient Index,$^{25}$ the Caregiver Contribution to Heart Failure SelfCare,${ }^{26}$ and the Caregiver Contribution to Self-Care of Heart Failure Index. ${ }^{27}$ Nevertheless, these tools are suitable for evaluating informal CC to self-care in ostomy and heart failure, and not suitable for generic chronic illnesses or multimorbidity. Given the above situation, Vellone $e t$ $a l^{28}$ developed the Caregiver Contribution to Self-Care Chronic Illness Inventory (CC-SC-CII), a reliable tool for assessing informal CC to self-care of patients with chronic diseases. Although informal CC to self-care was important in enhancing self-care and patient outcomes, evidence is limited on the CC level to self-care of patients with chronic illnesses in China. Therefore, our study aims to cross-culturally adapt and validate of the CC-SC-CII in China. It can help health professionals regularly assess the self-care behaviours of care partners, identify individuals with insufficient care levels and implement tailored interventions. Additionally, the current study contributes to shared decision-making between caregivers and healthcare professionals, which will reduce the adverse effects of chronic disease and improve the quality of life of patients. Because the characteristics of a member of the patient-caregiver dyad can influence the self-care behaviours of the other, the tool also helps provide a dyadic perspective when measuring self-care behaviours of patients and their care partners, which contributes to understanding the processes of patients and care partners to self-care. Moreover, cross culturally adapting a tool used by other researchers could perform data aggregation and outcomes comparisons among different studies and populations.

\section{METHODS}

\section{Study design and participants}

A cross-sectional survey conducted in China. Our study was reported following the Strengthening the Reporting of Observational studies in Epidemiology statement. Adopting a convenience sampling, 301 caregivers were recruited from communities and institutions in Pingdingshan, Henan Province, China. Data were collected from September to November 2020. The inclusion criteria for caregivers were: (1) age $\geq 18$ years old; (2) the main and informal caregiver of the patients who had chronic obstructive pulmonary disease, diabetes mellitus, heart failure, hypertension, asthma, osteoporosis or kidney failure; (3) no communication barriers (deafness or blindness). Patients with cancer or dementia were excluded. In our study, the inclusion criteria and exclusion criteria were similar to the study by Vellone $e t a l,{ }^{28}$ testing psychometric characteristics of the CC-SC-CII.

\section{Instruments \\ Demographic characteristics}

The demographic characteristics of caregivers and care recipients included age, sex, marital status, education levels, religion, employment status, family income, medical history, and so on.

\section{The Caregiver Contribution to Self-Care Chronic IIIness Inventory}

The CC-SC-CII is a 19-item tool measuring informal CC to self-care behaviours in patients with chronic illness and contains three scales: the CC to self-care maintenance scale, the CC to self-care monitoring scale and the CC to self-care management scale. As reported, the CC-SC-CII was developed based on the SC-CII. ${ }^{10} 29$ The maintenance scale (seven items) asks the care partners to report the frequency that caregivers recommend the patient for the common behaviours seeking to maintaining physical and mental stability. The monitoring scale (five items) assesses the frequency that caregivers recommend patient to monitor signs and symptoms of chronic illness. The management scale (seven items) measures the frequency that care partners help to recognise or explain symptoms and respond to worsening symptoms of chronic disease. The tool can only be completed when patients report any clinical manifestations of the chronic illness to care partners.

The score range of each CC-SC-CII scale is between 0 and 100, and higher scores indicate better CC. The CC to selfcare maintenance scale and the CC to self-care monitoring scale are rated on a 5-point Likert scale from 1 (never) to 5 (always). In the CC to self-care management scale, one 
item which tests symptom recognition has seven response options and use a skip-pattern format. The item uses a 5-point Likert response format from 1 (not quickly) to 5 (very quickly) plus 2 additional responses. If the two options are chosen, the score for this item is not calculated into the total score because it means that the patient has no symptoms, so the caregiver could not recognise them. Another 1 item which measures treatment evaluation using a 5-point Likert response format from (not sure) 1 to 5 (very sure) plus 1 additional response. If the option is selected by care partners, the score for the item is not calculated into the total scale score. Other terms in management scale have a 5-point Likert response format from 1 (not likely) to 5 (very likely). A score of $\geq 70$ means the CC to self-care is adequate on each scale.

\section{Study procedure}

After the permission of the original author (Dr. Vellone), the CC-SC-CII was translated into Chinese following the recommended guideline for the cross-cultural adaptation. $^{30}$ We conducted the psychometric evaluation of CC-SC-CII according to the original COnsensus-based Standards for the selection of health Measurement INstruments checklist.31

\section{Translation procedure}

Forward translation

First, two independent bilingual translators translated the CC-SC-CII into Chinese. Both of them were fluent in English and Mandarin.

\section{Synthesis of the two translated versions}

After forward translation, the comparison between two translated Chinese versions of CC-SC-CII (C-CC-SC-CII) and the original scale was performed by a third translator. The researchers of this study discussed and resolved all ambiguities and inconsistencies.

\section{Back translation}

Two independent translators translated the C-CC-SC-CII back into English.

\section{Synthesis of the two back-translated versions}

Our research team clarified the wording, grammatical structure, meaning equivalence and relevance of the two back-translations. Some ambiguities and inconsistencies were referred to the translators for clarification. Additionally, we sent the integrated version to the original author for confirmation. According to the suggestions of the original author, we modified some expressions of some terms. After these, an initial C-CC-SC-CII was formed.

\section{Cultural adaptation process}

Evaluation of the conceptual and content equivalence of items for the C-CC-SC-CII

The Delphi survey was conducted. Six experts, including four associate professors and two professors in chronic disease nursing, were consulted. The Delphi survey included the initial C-CC-SC-CII, the assessment documentation for content equivalence (using the scale of 1 to 4 : $1=$ not relevant; $2=$ unable to the assess relevance; $3=$ relevant but needs minor alteration; $4=$ very relevant and succinct) and biographical information (age, education level, etc). Then, it was emailed to the six experts. Six experts all gave their comments and suggestions. These revision suggestions were discussed among our research group. After these procedures, a consensus was achieved and the pre-final C-CC-SC-CII was formed.

\section{Pilot testing}

The prefinal C-CC-SC-CII was pilot tested using a sample of 99 caregivers. Participants took about 3 min to fill out the scale. Especially, each participant was invited to use 'clear' or 'unclear' describing the instruction and items of the scale, and was asked to provide suggestions on how to make each item clearer. After all the procedures, the final C-CC-SC-CII was generated for psychometric evaluation.

\section{Psychometric evaluation}

Validity

The content validity of the tool was evaluated using the Content Validity Index of Items (I-CVI) and the Scale Content Validity Index-Average (S-CVI/Ave) based on the ratings of experts on the scale. Confirmatory factor analysis (CFA) is applicable to the case where the dimensions of the measured construct are determined, while exploratory factor analysis (EFA) is suitable for the situation where the dimensions of the measured construct are uncertain. ${ }^{32}$ The CC-SC-CII was developed according to the middle-range theory of self-care of chronic illness, and three dimensions of the scale were determined. Therefore, CFA was employed to test construct-related validity. The sample size of 100-400 was considered adequate, and 200 was considered most appropriate for CFA. ${ }^{33}$ To be similar to previous validation studies based on the middle-range theory of self-care of chronic illness, ${ }^{10} 272934$ three scale in the C-CC-SC-CII were carried out CFAs.

\section{Reliability}

Scale reliability was evaluated using omega coefficient, composite reliability or Cronbach's alpha. Item-total corrected correlation coefficients was employed to calculate items discrimination. The intraclass correlation coefficient (ICC) was employed to assess the test-retest reliability. The 1-week to 2-week intervals was recommended to measure the test-retest reliability. ${ }^{35}$

\section{Procedures of data collection}

Data were collected in two methods: on-site data collection and online data collection. Well-trained and eligible research assistants who were all nursing Ph.D. candidates collected data through face-to-face interviews. All participants were informed the research purpose and that the participation was voluntary and confidential. Additionally, the C-CC-SC-CII was completed twice by a sample to calculate the test-retest reliability. 


\section{Data analysis}

The SPSS V.24.0 was used to perform analyse data. Descriptive statistics were calculated to describe the characteristics of participants. CFA was conducted using AMOS 22.0, to assess the structural validity of the C-CC-SC-CII. Because the C-CC-SC-CII items were non-normal distributed, the maximum likelihood estimation for CFA was chosen to assess the model fit. The following goodness-of-fit (GFI) indices were used to evaluate the model: the standardised root mean square residual (SRMR) was 0.08 or lower; the root mean square error of approximation (RMSEA) was 0.06 or lower; and GFI, comparative fit index (CFI), and Tucker-Lewis index (TLI) values were 0.95 or higher. ${ }^{36}$ A value of factor loads higher than $|0.30|$ was considered acceptable. ${ }^{37}$ The value of scale reliability coefficient $\geq 0.7$ was deemed to be adequate. ${ }^{38}$ The correlation analysis was performed to assess item-total correlation, and the itemtotal correlation varying from 0.30 and 0.80 was acceptable. $^{39}$ For the test-retest reliability, the value of ICC ranging from 0.75 and 0.90 indicated good reliability. ${ }^{40}$ In terms of content validity, I-CVI $\geq 0.78$ and S-CVI $\geq 0.80$ were considered acceptable. ${ }^{41}$

\section{Patient and public involvement}

The experts, the translators and the caregivers were regarded as public. In the current study, the information of patients was provided by their caregivers. Therefore, no patients were involved in our study during the crosscultural adaptation process of this scale.

\section{RESULTS}

\section{Translation and cultural adaptation}

Some terms were revised in the development of C-CCSC-CII. In item 5, 17, 18, 'healthcare provider' was not easily understood by caregivers in Chinese culture; therefore, healthcare provider was specific with 'doctors or nurses'. Furthermore, the 'conditions' was changed to 'health conditions' in item 8. In our study, 99 main caregivers were randomly selected for a pre-survey to determine whether the scale could be correctly understood and filled out correctly. The results of the pretest indicated that the caregivers understood the items easily and took about $2-4 \mathrm{~min}$ to fill out the scale. The origin CC-SC-CII scale and translated and adapted version are shown in table 1.

\section{Characteristics of caregivers and care recipients}

Three hundred and twenty-five main caregivers were approached for this study. However, 301 main caregivers (participation rate $=92.6 \%$ ) completed the survey. Their age was between 18 and 83 years (mean=42.61, $\mathrm{SD}=11.59)$. Most caregivers lived in the city $(50.2 \%)$, had high school/specialised secondary school education levels $(35.5 \%)$, were employed $(72.4 \%)$, did not have religion $(82.1 \%)$ and married $(79.40 \%)$. The age of care recipients was between 50 and 73 years (mean $=59.58$, $\mathrm{SD}=19.05$ ). The $48.5 \%$ of care recipients had one chronic disease. The sociodemographic information of the participants is presented in table 2 .

\section{Psychometric analysis \\ Validity \\ Content validity}

The content validity of the primary version of the C-CCSC-CII was assessed through the expert consultation. The current study indicated that the content validity of C-CCSC-CII was excellent with I-CVI being between 0.833 and 1.00 and the S-CVI being 0.991.

\section{Construct validity}

The CC to self-care maintenance scale included 'health promoting behaviors' dimension and 'illness-related behaviors dimension. For C-CG to self-care maintenance scale, we identified two factors and specified the correlations of residuals between item 2 and item 4 in CFA. The result of CFA testing the two-factor structure had unsatisfactory fit indices : $\chi^{2} / \mathrm{df}=9.278, \mathrm{p}=0.000 ; \mathrm{GFI}=0.892$, AGFI $=0.749, \mathrm{CFI}=0.822 ;$ TLI $=0.689 ; \mathrm{RMSEA}=0.166(90 \%$ CI 0.139 to $0.195 ; \mathrm{p}=0.000$ ); and $\mathrm{SRMR}=0.078$ (figure 1 ). To explore the best factor structure, EFA was used. The value of the Kaiser-Meyer-Olkin (KMO) test was 0.779, and Bartlett's test of sphericity was significant $(\mathrm{p}=0.000)$, which indicated that the 7-item C-CG to self-care maintenance scale was adequate for EFA. The oblique rotation method was used to extract two factors with eigenvalues $>1.0,{ }^{42}$ and the cumulative variance contribution rate of the two factors was $61.353 \%$. Therefore, the maintenance scale was a two-factor structure, which were composed of illness-related behaviours (items 1, 5, 6) dimension and health promoting behaviours dimension (items 2, 3, 4, 7) in the context of Chinese culture.

The CC to self-care monitoring scale had a factor structure containing 5 items, and the residuals of item 8 and item 10 covaried; therefore, we also specified this in our study. The result of CFA testing this 1-factor model found that the model did not fit well: $\chi^{2} / \mathrm{df}=4.057$, $\mathrm{p}=0.000 ; \mathrm{GFI}=0.978$, AGFI=0.918, CFI=0.984; TLI=0.959; RMSEA $=0.101$ (90\% CI 0.053 to $0.154 ; \mathrm{p}=0.041)$; and $\mathrm{SRMR}=0.0222$. The modification indices suggested estimation of the error covariances between item 8 and item 11 and between item 11 and item 12. When we respecified the model to include these error covariances, model fit improved: $\chi^{2} / \mathrm{df}=2.240, \mathrm{p}=0.081$; CFI=0.995; TLI=0.983; RMSEA $=0.064(90 \%$ CI 0.000 to $0.131 ; \mathrm{p}=0.284)$; and $\mathrm{SRMR}=0.0141$ (figure 2).

The CC to self-care management scale had seven items, reflecting two dimensions: 'autonomous behavior' and 'consulting behaviors'. For C-CC to self-care management scale, we specified a two-factor model and the correlations of residuals between items 13 and 19, between items 14 and 15 . When we specified the two dimensions at CFA, the model did not fit well : $\chi^{2} / \mathrm{df}=6.538, \mathrm{p}=0.000$; GFI=0.903, AGFI $=0.840, \mathrm{CFI}=0.939 ; \mathrm{TLI}=0.884 ; \mathrm{RMSEA}=0.136(90 \%$ CI 0.107 to $0.167 ; \mathrm{p}=0.00$ ), and $\mathrm{SRMR}=0.0441$ (figure 3). Similarly, to identify the best factor structure, EFA was 
Table 1 The origin Caregiver Contribution to Self-Care Chronic Illness Inventory (CC-SC-CII) scale items and final C-CC-SCCll items

\begin{tabular}{l}
\hline Original CC-SC-CII scale items \\
1.Make sure to get enough sleep \\
2.Do physical activity (eg, take a brisk walk, use the stairs) \\
3.Do something to relieve stress (eg, medication, yoga, music)
\end{tabular}

Translated and adapted version

1.Ensure enough sleep

保证充足的睡眠

2.Attend physical activities (eg, brisk walking, climb stairs)

进行体育活动(例如, 快走, 走楼梯)

3.Do something to relieve stress (eg, drugs, Yoga, music)

做些缓解压力的事情 (例如药物, 瑜伽, 音乐)

4.Eat a special diet

5.See your healthcare provider for routine healthcare

4.Eat a special diet

吃特定的食物 (例如, 低脂饮食, 低盐饮食)

5.Acquire health guidance from doctors or nurses

从医生或者护士处获取健康指导

6. Take prescribed medicines without missing a dose

6. Take medicine as prescribed by the doctor and never miss a dose

遵医嘱服药, 绝不漏服

7.Try to avoid getting sick (eg, influenza shot, wash the hands)

7.Try to avoid getting sick (eg, get a influenza shot, wash your hands)

尽量避免生病 (例如, 注射流感疫苗, 洗手)

8.Monitor the conditions of the person you care for?

8.Monitor the health conditions of the person you care for?

监测您照顾的人的健康状况?

9.Monitor for medication side-effects of the person you care for?

9.Monitor the medication side effects of the person you care for?

监测您照顾的人的用药副作用?

10.Pay attention to changes in how the person you care for feels?

10.Pay attention to the changes in the feelings of the person you care for?

关注您照顾的人感觉的变化?

11.Monitor whether the person you care for tires more than usual doing normal activities?

12.Monitor for symptoms of the person you care for?

Original CC-SC-CIl scale items

11.Monitor whether the person you care for is more tired than usual in doing normal activities?

监测您照顾的人在进行正常活动时是否比平时更疲志?

12.Monitor the symptoms of the person you care for?

Translated and Adapted Version

监测您照顾的人的症状?

13. How quickly did you recognise them as symptoms of the illness he/she suffers from?

\begin{tabular}{|c|c|}
\hline & 您多快识别这些是他/她所患疾病的症状？ \\
\hline \multirow{2}{*}{$\begin{array}{l}\text { 14.Change what he/she eats or drinks to make the symptom decrease or } \\
\text { go away? }\end{array}$} & 14.Change what he/she eats or drinks to get symptoms decreased or disappeared? \\
\hline & 改变他/她摄入的食物或饮料, 使症状减轻或消失？ \\
\hline \multirow[t]{2}{*}{ 15.Change the activity level (eg, slow down, rest)? } & 15.Change activity level (eg, slow down, take a rest)? \\
\hline & 改变活动水平 (例如 , 慢下来, 休息) ？ \\
\hline \multirow[t]{2}{*}{ 16.Take a medicine to make the symptom decrease or go away? } & 16.Take a medicine to get symptoms decreased or disappeared? \\
\hline & 服药使症状减轻或消失？ \\
\hline \multirow[t]{2}{*}{ 17.Tell the healthcare provider about the symptom at the next office visit? } & 17.Tell the doctor/nurse about your symptoms at the next visit? \\
\hline & 在下次就诊时告诉医生或者护士症状？ \\
\hline \multirow[t]{2}{*}{ 18.Call the healthcare provider for guidance? } & 18.Call doctors/nurses for guidance? \\
\hline & 打电话给医生或者护士寻求指导？ \\
\hline \multirow[t]{2}{*}{ 19.Did the treatment you used make feel the person you care for better? } & 19.Did the treatment you adopted make the person you care for feel better? \\
\hline & 您采用的治疗方法是否让您照顾的人感觉好些了？ \\
\hline
\end{tabular}

performed. The KMO index was 0.869 , and the results of the Bartlett sphericity test were also statistically significant $(\mathrm{p}=0.000)$, which meant that the 7-item management scale was also suitable for EFA. Similarly, the use of the oblique rotation method extracted one factor with eigenvalues $>1.0,{ }^{42}$ and the cumulative variance explained by the factor was $58.138 \%$. Therefore, in the context of
Chinese culture, the management scale may have a factor structure.

Item-total correlation and reliability

Table 3 shows the item-total correlation. Each item was correlated to total score of the three scales (range, 0.5920.870) (table 3). Regarding to the reliability, because 
Table 2 Characteristics of the included informal caregivers and care recipients $(n=301)$

\begin{tabular}{|c|c|c|c|c|}
\hline & \multicolumn{2}{|c|}{ Informal caregiver } & \multicolumn{2}{|c|}{ Care recipient } \\
\hline Characteristics & \multicolumn{2}{|c|}{$\mathrm{M} \pm \mathrm{SD} / \mathrm{N}(\%)$} & \multicolumn{2}{|c|}{$M \pm S D / N(\%)$} \\
\hline \multicolumn{5}{|l|}{ Gender } \\
\hline Man & 139 & 46.2 & 162 & 53.8 \\
\hline Woman & 162 & 53.8 & 139 & 46.2 \\
\hline Age & \multicolumn{2}{|c|}{$42.61 \pm 11.59$} & \multicolumn{2}{|c|}{$59.58 \pm 19.05$} \\
\hline
\end{tabular}

\section{Education level}

\begin{tabular}{lll}
$\begin{array}{l}\text { Junior school or } \\
\text { below }\end{array}$ & 71 & 23.6 \\
$\begin{array}{l}\text { High school/ } \\
\text { specialised } \\
\text { secondary school }\end{array}$ & 107 & 35.5 \\
$\begin{array}{l}\text { Specialty/Bachelor } \\
\begin{array}{l}\text { Postgraduate or } \\
\text { above }\end{array}\end{array}$ & 87 & 28.9 \\
\hline
\end{tabular}

\begin{tabular}{|c|c|c|}
\hline \multicolumn{3}{|l|}{ Residence } \\
\hline City & 151 & 50.2 \\
\hline Town & 105 & 34.9 \\
\hline Countryside & 45 & 15 \\
\hline \multicolumn{3}{|c|}{ Employment status } \\
\hline Employed & 218 & 72.4 \\
\hline Unemployed & 83 & 27.6 \\
\hline \multicolumn{3}{|l|}{ Religion } \\
\hline Yes & 54 & 17.9 \\
\hline No & 247 & 82.1 \\
\hline \multicolumn{3}{|l|}{ Marital status } \\
\hline $\begin{array}{l}\text { Single/never } \\
\text { married }\end{array}$ & 41 & 13.62 \\
\hline Married & 239 & 79.40 \\
\hline Divorced & 13 & 4.32 \\
\hline Widowed & 8 & 2.66 \\
\hline
\end{tabular}

Average income ( $¥$, monthly)

\begin{tabular}{lrr|}
$<2000$ & 53 & 17.6 \\
\hline $2000-4000$ & 136 & 45.2 \\
$4001-6000$ & 56 & 18.6 \\
$>6000$ & 56 & 18.6
\end{tabular}

\section{Relationship with care recipient}

\begin{tabular}{lcc} 
Spouse/partner & 49 & 16.3 \\
\hline Parents & 77 & 25.6 \\
Child & 59 & 19.6 \\
Sister/brother & 17 & 5.6 \\
Friend & 24 & 8 \\
Son-/daughter-in- & 15 & 5 \\
law & & \\
Other & 60 & 19.9
\end{tabular}

Other $\quad 60 \quad 19.9$

\section{Cohabitation with care recipient}

$\begin{array}{lll}\text { Yes } & 157 & 52.2 \\ \text { No } & 144 & 47.8\end{array}$

Continued
Table 2 Continued

Informal caregiver Care recipient

N.of chronic

illnesses

\begin{tabular}{lc} 
One & 14648.5 \\
\hline Two & 8427.9 \\
\hline Three & 4815.9 \\
\hline Four & 155 \\
>Four & 82.7
\end{tabular}

Cronbach's alpha assumes that the items satisfy a unidimensional factor structure, the global reliability index for multidimensional scales was employed to assess reliability coefficient of the C-CC to self-care maintenance scale with two dimensions ${ }^{43}$ which was 0.792 in this scale. In the monitoring scale, given that the factor loadings were unequal and there were covariances among residuals, we chose Raykov's composite reliability coefficient to do the analysis ${ }^{43}$ and the value was 0.880 . The reliability of the management scale was measured with a Cronbach's alpha value, which was 0.870 . The above reliability results indicated acceptable and satisfactory internal consistency. For test-retest reliability, after 2 weeks, 20 caregivers completed the follow-up assessment. Test-retest reliability showed the range of ICC was between 0.728 and 0.783 , indicating that the three scales of C-CC-SC-CII have satisfactory stability.

\section{DISCUSSION}

With the ageing population, increasing healthcare costs and the lifelong impact of chronic diseases, informal care is becoming more and more important. ${ }^{44}$ Informal

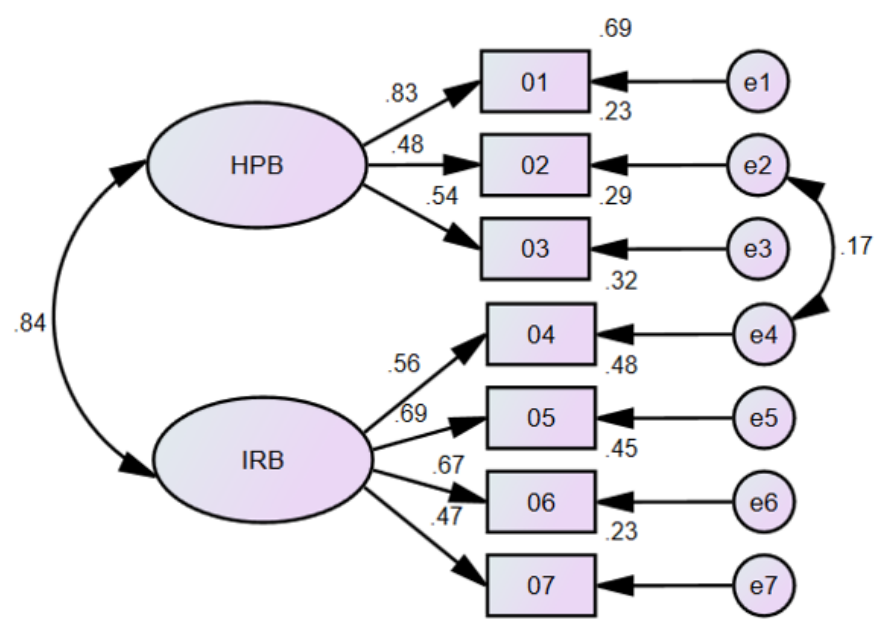

Figure 1 Confirmatory factor analysis of the C-CC to selfcare maintenance scale. The numbers near the 1-headed arrows are factor loading coefficients; the numbers near the 2-headed arrows are correlation coefficients; the numbers aiming to the items through a 1-headed arrow are residual variances. HPB, health promoting behaviours; IRB, illnessrelated behaviours; 01, item 1 . 


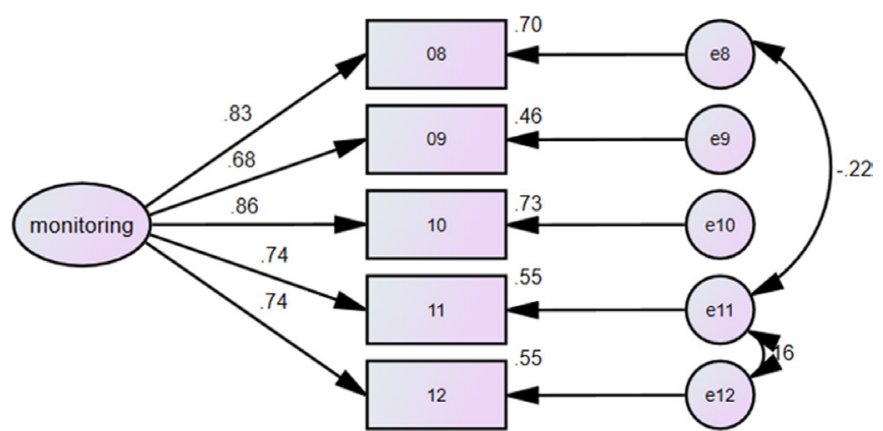

Figure 2 Confirmatory factor analysis of the C-CC to selfcare monitoring scale. The numbers near the 1 -headed arrows are factor loading coefficients; the numbers near the 2-headed arrows are correlation coefficients; the numbers aiming to the items through a 1-headed arrow are residual variances. 08 , item 8 .

caregivers display a crucial role in taking care of their care recipients with chronic diseases and there is evidence that informal CC to self-care behaviours can enhance patient health outcomes. Therefore, it is essential to find an evidence-based, convenient, and practical measurement to quantity informal CC level in China, which could help healthcare professionals quickly identify informal CC level and design and implement more targeted interventions. Furthermore, the tool can provide a dyadic perspective when studying the self-care process of patients and their care partners. Moreover, China lacks a standardised tool to quantity informal CC level, so our study sought to cross culturally adapt and validate the CC-SC-CII into Chinese. The present study demonstrated that the C-CC-SC-CII was a reliable measurement.

The translation and adaptation of CC-SC-CII were undertaken strictly following the established guideline. ${ }^{30}$ When compared with the CC-SC-CII, four items were revised in the C-CC-SC-CII. In China, to make 'healthcare provider' easier to be understood by caregivers,

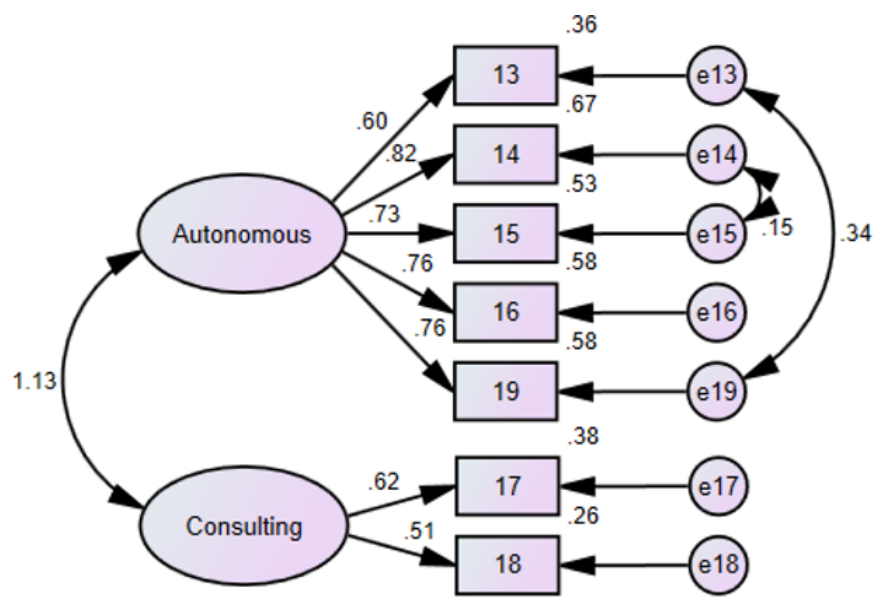

Figure 3 Confirmatory factor analysis of the C-CC to selfcare management scale. The numbers near the 1-headed arrows are factor loading coefficients; the numbers near the 2-headed arrows are correlation coefficients; the numbers aiming to the items through a 1-headed arrow are residual variances. 13, item 13.

\begin{tabular}{|c|c|c|}
\hline Items & Item-total correlations & $P$ value \\
\hline 1 & $0.757^{\star *}$ & $<0.001$ \\
\hline 2 & $0.633^{\star *}$ & $<0.001$ \\
\hline 3 & $0.694^{\star *}$ & $<0.001$ \\
\hline 4 & $0.683^{\star \star}$ & $<0.001$ \\
\hline 5 & $0.655^{\star *}$ & $<0.001$ \\
\hline 6 & $0.617^{\star \star}$ & $<0.001$ \\
\hline 7 & $0.592^{\star *}$ & $<0.001$ \\
\hline 8 & $0.842^{* *}$ & $<0.001$ \\
\hline 9 & $0.786^{\star \star}$ & $<0.001$ \\
\hline 10 & $0.870^{\star *}$ & $<0.001$ \\
\hline 11 & $0.801^{\star \star}$ & $<0.001$ \\
\hline 12 & $0.827^{\star \star}$ & $<0.001$ \\
\hline 13 & $0.748^{* *}$ & $<0.001$ \\
\hline 14 & $0.835^{\star \star}$ & $<0.001$ \\
\hline 15 & $0.778^{\star *}$ & $<0.001$ \\
\hline 16 & $0.731^{\star *}$ & $<0.001$ \\
\hline 17 & $0.679^{\star *}$ & $<0.001$ \\
\hline 18 & $0.662^{\star \star}$ & $<0.001$ \\
\hline 19 & $0.844^{\star *}$ & $<0.001$ \\
\hline
\end{tabular}

CC-SC-CII, Caregiver Contribution to Self-Care Chronic Illness Inventory.

'healthcare provider' was revised as 'doctors or nurses' in items $5,17,18$. In addition, in item 8 , 'conditions' referred to 'health conditions'. Therefore, 'conditions' was revised to 'health conditions'. Moreover, the scale proved understandable and needed to take about 2-4 min to complete. We sought the advice of Dr. Vellone, the developer of CC-SC-CII, on the translation and crosscultural adaptation.

In general, the scale structure of the C-CC-SC-CII was different from the original Italian scale, although the C-CC-SC-CII also included three scales and 19items. In regard to the C-CC to self-care maintenance scale, we identified two factors through EFA: the health promoting behaviour dimension (items 2, 3, 4, 7) and the illnessrelated behaviour dimension (items $1,5,6$ ), which was different from the origin scale. We were interested to find that 'ensure enough sleep' was an 'illness-related behavior' in our study. Reallocation of item 1 to 'illnessrelated behavior' may be associated with adverse health outcomes from sleep deprivation such as multiple acute and chronic diseases, which led to increased mortality and morbidity of cardiovascular diseases. ${ }^{45}$ The phenomenon illustrated that people seemed to view enough sleep as a necessity rather than an option. Therefore, from a clinical point of view, this reallocation is plausible. In addition, in our study, washing the hands was a kind of health promoting behaviours to prevent diseases in China. ${ }^{46}$ Ataee $e t a l^{47}$ also showed that handwashing was crucial for health promotion and infections control. Therefore, the 
reallocation of item 7 to the health promoting behaviour was not surprising. However, given the limited sample size, this model was not validated in the present study. Future studies need verify the model in a larger sample to find the maintenance model suitable for China.

The C-CC to self-care monitoring scale had a factor structure in CFA. We specified the covariance between residuals of items 8 and 11, and between items 11 and 12 to fit the data, when Vellone $e t a t^{28}$ specified the covariance between residuals of item 8 and item 10 . The existence of covariance suggested that our care partners usually connected these monitoring behaviours. Due to the nursing shortage, it is very difficult for a nurse to take care of a patient in China. Therefore, our caregivers often were taught by healthcare professionals to monitor the health conditions of care recipients, which included glycaemic indices, blood pressure, temperature, fatigue, disease-related symptoms, and so on. These conditions were usually monitored by caregivers together in the context of Chinese situation. In these contexts, these covariances are plausible.

As for the C-CC to self-care management scale, one dimension was found. The original CC to self-care management scale included two dimensions: autonomous behaviours and consulting behaviours. Autonomous behaviours refer to those behaviours that individuals choose either congenitally or based on previous experience when they have symptoms. Consulting behaviours usually refer to those behaviours recommended by others. ${ }^{10}$ In our sample, our interpretation of a unidimensional model is that in Chinese cultural background, caregivers often consulted health providers and listen to their advice to care for patients with chronic disease such as making them change activity level and take medicine. As a result, autonomous behaviours and consulting behaviours are closely related. This was our situation. We need further research to verify this factor structure.

The reliability evaluation showed that the C-CC-SC-CII was an acceptable instrument in this population. The reliability coefficient of three scales was $0.792,0.880$ and 0.870 . Test-retest reliability of three scales showed ICC ranged from 0.728 to 0.783 . This feature is important when the C-CC-SC-CII is employed to assess the effectiveness of interventions to increase CC level. Additionally, this study also showed that all the items had adequate discrimination. The psychometric characteristics of the C-CC-SC-CII supported the stability and internal consistency of the instrument.

However, the current study has some limitations. First, our study is limited by the choice of the convenience sampling, which may not be representative enough. Second, there were no adequate instruments for evaluating concurrent validity. Although the C-CC-SC-CII and SC-CII measured self-care, one evaluated the CC to selfcare behaviours of individuals with chronic illnesses, and the other assessed self-care behaviours of patients with chronic illnesses. The concurrent validity of CC-SC-CII was measured with SC-CII in Vellone $e t a l^{28}$ study. Therefore,
SC-CII can be considered to measure the concurrent validity of C-CC-SC-CII. ${ }^{10}$ However, there is no validated Chinese version of SC-CII. Hence, further studies should consider evaluating the correlation between the Chinese version of SC-CII and C-CC-SC-CII to calculate the concurrent validity.

\section{CONCLUSION}

We have identified the 19-item C-CC-SC-CII as a validated scale in the measurement of CC among Chinese caregivers of patients with chronic diseases. The scale provides a simple and rapid solution to assess the self-care process carried out by the caregivers, which is important, given the increasing rate of chronic disease. The scale also allows health professionals to understand the informal CC level to improve care process and patient outcomes better. The scale needs to be further verified among a larger sample.

\section{Author affiliations}

${ }^{1}$ Nursing Department, Affiliated Sir Run Run Shaw Hospital, Zhejiang University School of Medicine, Hangzhou, Zhejiang, China

${ }^{2}$ Nursing Department, Second Affiliated Hospital, Zhejiang University School of Medicine, Hangzhou, Zhejiang, China

${ }^{3}$ School of Nursing, Zhejiang University School of Medicine, Hangzhou, Zhejiang, China

${ }^{4}$ School of Nursing, Shanghai Jiao Tong University School of Nursing, Shanghai, China

${ }^{5}$ Thyroid\&breast surgery, Chongqing Medical University Affiliated Second Hospital, Chongqing, China

\section{Twitter Nianqi Cui @NianqiC}

Acknowledgements The authors would like to express our sincere appreciation to the experts, the original author of the scale and the students, who participated in the study.

Contributors DDC conducted the analysis plan, performed the data collection and analysis, interpreted the results and wrote the manuscript. $\mathrm{HZ}$ and XW confirmed and modified the back-translated version of the C- CC-SC-CII. NL, NC, LT, JS and DW reviewed the manuscript and modified important content. NL and NC carried out data collection and analysis. ZY supervised the study, conducted the study design, contacted experts to translate and revise the manuscript, led the data collection, and approved the final draft. The manuscript was read and approved by all authors.

Funding This study was funded by the National Office for Philosophy and Social Sciences of China (20BGL275); This study was supported by the National Natural Science Foundation of China (72004193).

\section{Competing interests None declared.}

Patient and public involvement Patients and/or the public were not involved in the design, or conduct, or reporting, or dissemination plans of this research.

\section{Patient consent for publication Not required.}

Ethics approval Ethics for the study was approved by the Sir Run Run Shaw Hospital, Zhejiang University School of Medicine (20200115-31). All caregivers voluntarily participated and signed informed consent forms and their information would remain confidential.

Provenance and peer review Not commissioned; externally peer reviewed.

Data availability statement Data are available upon reasonable request.

Open access This is an open access article distributed in accordance with the Creative Commons Attribution Non Commercial (CC BY-NC 4.0) license, which permits others to distribute, remix, adapt, build upon this work non-commercially, and license their derivative works on different terms, provided the original work is properly cited, appropriate credit is given, any changes made indicated, and the use is non-commercial. See: http://creativecommons.org/licenses/by-nc/4.0/. 


\section{ORCID iDs}

Nianqi Cui http://orcid.org/0000-0002-7963-4887

Xiyi Wang http://orcid.org/0000-0001-9908-2760

Zhihong Ye http://orcid.org/0000-0001-6947-3330

\section{REFERENCES}

1 Nikolich-Žugich J, Goldman DP, Cohen PR, et al. Preparing for an aging world: engaging Biogerontologists, geriatricians, and the Society. J Gerontol A Biol Sci Med Sci 2016;71:435-44.

2 Suls J, Green PA, Boyd CM. Multimorbidity: implications and directions for health psychology and behavioral medicine. Health Psychol 2019;38:772-82.

3 Yoon J, Zulman D, Scott JY, et al. Costs associated with multimorbidity among Va patients. Med Care 2014;52 Suppl 3:S31-6.

4 Schmidt H. Chronic Disease Prevention and Health Promotion, In: Barrett DH, ed. Public health ethics: cases spanning the globe. Cham: Springer International Publishing, 2016: 137-76.

5 OEG. Certain social problems caused by chronic illnesses. Selçuk University J Inst Soc Sci 2019;42.

6 GBD 2017 Causes of Death Collaborators. Global, regional, and national age-sex-specific mortality for 282 causes of death in 195 countries and territories, 1980-2017: a systematic analysis for the global burden of disease study 2017. Lancet 2018;392:1736-88.

7 Benziger CP, Roth GA, Moran AE. The global burden of disease study and the preventable burden of ncd. Glob Heart 2016;11:393-7.

8 Promotion, N.C.F.C. Chronic disease overview, 2017.

9 De Maria M, Matarese M, Strömberg A, et al. Cross-Cultural assessment of the self-care of chronic illness inventory: a psychometric evaluation. Int J Nurs Stud 2021;116:103422.

10 Riegel B, Barbaranelli C, Sethares KA, et al. Development and initial testing of the self-care of chronic illness inventory. J Adv Nurs 2018;74:2465-76.

11 Riegel B, Jaarsma T, Strömberg A. A middle-range theory of self-care of chronic illness. ANS Adv Nurs Sci 2012;35:194-204.

12 Cannon D, Buys N, Sriram KB, et al. The effects of chronic obstructive pulmonary disease self-management interventions on improvement of quality of life in COPD patients: a meta-analysis. Respir Med 2016;121:81-90.

$13 \mathrm{He}$ X, Li J, Wang B, et al. Diabetes self-management education reduces risk of all-cause mortality in type 2 diabetes patients: a systematic review and meta-analysis. Endocrine 2017;55:712-31.

14 Dickson VV, Buck H, Riegel B. Multiple comorbid conditions challenge heart failure self-care by decreasing self-efficacy. Nurs Res 2013;62:2-9.

15 Buck HG, Dickson VV, Fida R, et al. Predictors of hospitalization and quality of life in heart failure: a model of comorbidity, self-efficacy and self-care. Int J Nurs Stud 2015;52:1714-22.

16 Wu S-FV, Lee M-C, Liang S-Y. Effectiveness of a self-efficacy program for persons with diabetes: a randomized controlled trial. Nurs Health Sci 2011;13:no-43.

17 Smith ML, Bergeron CD, Riggle SD, et al. Self-Care difficulties and reliance on support among vulnerable middle-aged and older adults with chronic conditions: a cross-sectional study. Maturitas 2017;104:1-10.

18 lovino P, Lyons KS, De Maria M, et al. Patient and caregiver contributions to self-care in multiple chronic conditions: a multilevel modelling analysis. Int J Nurs Stud 2021;116:p. 103574..

19 Riegel B, Moser DK, Buck HG, et al. Self-Care for the prevention and management of cardiovascular disease and stroke: a scientific statement for healthcare professionals from the American heart association. J Am Heart Assoc 2017;6. doi:10.1161/ JAHA.117.006997. [Epub ahead of print: 31 Aug 2017].

20 Kang X, Li Z, Nolan MT. Informal caregivers' experiences of caring for patients with chronic heart failure: systematic review and metasynthesis of qualitative studies. $J$ Cardiovasc Nurs 2011;26:386-94.

21 Durante A, Greco A, Annoni AM, et al. Determinants of caregiver burden in heart failure: does caregiver contribution to heart failure patient self-care increase caregiver burden? Eur J Cardiovasc Nurs 2019;18:691-9.

22 Durante A, Paturzo M, Mottola A, et al. Caregiver contribution to self-care in patients with heart failure: a qualitative descriptive study. J Cardiovasc Nurs 2019;34:E28-35.

23 Krug K, Miksch A, Peters-Klimm F, et al. Correlation between patient quality of life in palliative care and burden of their family caregivers: a prospective observational cohort study. BMC Palliat Care 2016;15:4.

24 Farquhar M. Carers and breathlessness. Curr Opin Support Palliat Care 2017;11:165-73.

25 Villa G, Vellone E, Sciara S, et al. Two new tools for self-care in ostomy patients and their informal caregivers: psychosocial, clinical, and operative aspects. Int J Urol Nurs 2019;13:23-30.

26 Buck HG, Harkness K, Ali MU, et al. The caregiver contribution to heart failure self-care (CACHS): further psychometric testing of a novel instrument. Res Nurs Health 2017;40:165-76.

27 Vellone E, Barbaranelli C, Pucciarelli G, et al. Validity and reliability of the caregiver contribution to self-care of heart failure index version 2 $J$ Cardiovasc Nurs 2020;35:280-90.

28 Vellone E, Lorini S, Ausili D, et al. Psychometric characteristics of the caregiver contribution to self-care of chronic illness inventory. J Adv Nurs 2020;76:2434-45.

29 Riegel B, Barbaranelli C, Carlson B, et al. Psychometric testing of the revised self-care of heart failure index. J Cardiovasc Nurs 2019;34:183-92.

30 Sousa VD, Rojjanasrirat W. Translation, adaptation and validation of instruments or scales for use in cross-cultural health care research: a clear and user-friendly guideline. J Eval Clin Pract 2011;17:268-74.

31 Mokkink LB, de Vet HCW, Prinsen CAC, et al. COSMIN risk of bias checklist for systematic reviews of patient-reported outcome measures. Qual Life Res 2018;27:1171-9.

32 TA B. Confirmatory factor analysis for applied research. NewYork: The Guilford Press, 2015.

33 Hair BWBB. Multivariate data analysis. 7 edn. Upper Saddle River, NJ: Pearson Prentice Hal, 2010.

34 Matarese M, Clari M, De Marinis MG, et al. The self-care in chronic obstructive pulmonary disease inventory: development and psychometric evaluation. Eval Health Prof 2020;43:50-62.

35 Terwee CB, Bot SDM, de Boer MR, et al. Quality criteria were proposed for measurement properties of health status questionnaires. J Clin Epidemiol 2007;60:34-42.

$36 \mathrm{Hu}$ Li-tze, Bentler PM. Cutoff criteria for fit indexes in covariance structure analysis: conventional criteria versus new alternatives. Struct Equ Model 1999;6:1-55.

37 Tabachnick BGFL. Using multivariate statistics. Boston, MA: Pearson Education, 2007.

38 Bagozzi RP, Yi Y. Specification, evaluation, and interpretation of structural equation models. J Acad Mark Sci 2012;40:8-34.

39 Thoyre SM, Pados BF, Park J, et al. The pediatric eating assessment tool: factor structure and psychometric properties. J Pediatr Gastroenterol Nutr 2018;66:299-305.

40 Koo TK, Li MY. A guideline of selecting and reporting intraclass correlation coefficients for reliability research. J Chiropr Med 2016;15:155-63.

41 Lynn MR. Determination and quantification of content validity. Nurs Res 1986;35:382???386-5.

42 Rattray J, Jones MC. Essential elements of questionnaire design and development. J Clin Nurs 2007;16:234-43.

43 Raykov T. Scale construction and development using structural equation modeling. New York: The Guilford Press, 2013.

$44 \mathrm{Kim} \mathrm{H}$, Lee S, Cheon J, et al. A comparative study to identify factors of caregiver burden between baby boomers and post baby boomers: a secondary analysis of a US online caregiver survey. BMC Public Health 2018;18:579.

45 Liu H, Chen A. Roles of sleep deprivation in cardiovascular dysfunctions. Life Sci 2019;219:231-7.

46 Xiangyun FU. Evaluation of the health promotion effect on hand hygiene in medical staff. Modern Prev Med 2014;7.

47 Ataee RA, Ataee MH, Mehrabi Tavana A, et al. Bacteriological aspects of hand washing: a key for health promotion and infections control. Int J Prev Med 2017;8:16. 